\title{
Biomechanical Evaluation of two different types of Interbody Cages in Posterior Lumbar Interbody Fusion
}

\author{
Muhammad Hazli Mazlan ${ }^{1,2}$, Mitsugu Todo ${ }^{3}$, Ida Laila ahmad ${ }^{1,2}$, Hiromitsu Takano ${ }^{4}$, Ikuho Yonezawa ${ }^{4}$ \\ ,Abdul Halim Abdullah ${ }^{5}$, Muhammad Hilmi Jalil ${ }^{6}$ and Nur Dalilah Diyana Nordin ${ }^{7}$ \\ ${ }^{1}$ Microelectronics and Nanotechnolgy-Shamsuddin Research Center, Universiti Tun Hussein Onn Malaysia, \\ 86400 Parit Raja, Batu Pahat, Malaysia,mhazli@uthm.edu.my \\ ${ }^{2}$ Faculty of Electrical and Electronic Engineering, Universiti Tun Hussein Onn, Malaysia, 86400 Parit Raja, Batu \\ Pahat, Malaysia. \\ ${ }^{3}$ Research Institute of Applied Mechanics, Kyushu University, Japan \\ ${ }^{4}$ Department of Orthopedic Surgery, Juntendo University, Japan \\ ${ }^{5}$ Department of Mechanical Engineering, Universiti Teknologi Mara, Malaysia \\ ${ }^{6}$ Department of Mechanical Engineering, Universiti Malaysia Pahang, Malaysia \\ ${ }^{7}$ Center of Information Technology, Universiti Tun Hussein Onn, 86400 Parit Raja, Batu Pahat, Malaysia
}

\begin{abstract}
Posterior lumbar interbody fusion (PLIF) related complications such as cage instability, cage subsidence and pedicle screws loosening are among the most prevalent cases reported postoperatively. These conditions are highly related to mechanical factors (PLIF design and material), patient health condition as well as activities conducted by the patient after undergone the surgery. Latest advancement on PLIF technology has created a new technique that allows the application of unilateral cage insertion in an oblique orientation. This solution has potentially overcome the problem related to an unintended mechanical and clinical shortcoming, provided that a bilateral posterior instrumentation (PI) is instrumented to the construct and the cage is fabricated from a material that is closely imitate the modulus elasticity of the cortical bone. In order to prove these statements, an image based finite element analysis (FEA) was conducted to assess the phenomena of cage subsidence and screw loosening by examining the stress profile on the cage construct and the vertebral bodies. Obliquely-placed unilateral PLIF with PI showed the most promising results. It showed the most minimal stress distortion at cage-endplate and pedicle screw-bone interface. In conclusion, the selection of a biocompatible cage material is the most crucial factors that has to be considered in achieving biomechanical superiority in PLIF surgery.
\end{abstract}

Key words: Drucker-Prager Stress, Finite Element Analysis, Posterior Instrumentation, Posterior Lumbar Interbody Fusion, Spine.

\section{INTRODUCTION}

The primary function of the posterior lumbar interbody fusion (PLIF) with assistive posterior instrumentation (PI) is to improve structural stability of the motion segment, to assist fusion and to eliminate severe back pain [1]. This surgical procedure is adopted when severe degenerative disc disease with disc space collapse is identified. Other conditions that may also require spinal fusion includes spinal disc herniation, vertebral fracture, scoliosis, spondylosis, kyphosis and any condition that causes instability of the spine. This treatment has been widely accepted as 80,000 interbody fusions were successfully implanted in the year of 1995 to 1999 [2].

Cage is initially fabricated from either medical grade titanium alloy or stainless steel. Latest advancement in medical research has discovered a new medical grade polyetheretherketone (PEEK) material. This material shows better radiolucency if compared to the preceding materials that improves the visibility thus improving the accuracy to locate the cage on the targeted areas. In addition, it is also more compatible to bone as the stiffness of the material $(\mathrm{E}=$ $3.6 \mathrm{GPa})$ is nearer to that of cortical bone $(\mathrm{E}=12 \mathrm{GPa})$. This feature can significantly reduce the stress shielding effect that is associated to titanium alloy $(\mathrm{E}=110 \mathrm{GPa})$ [3]. As the research in medical expanding, carbon fiber reinforced polymers (CF-P) has been introduced to improve the quality of PEEK material. This material has shown high fusion rates and satisfactory clinical outcomes. However, the stiffness factor has undermined the performance of this material over PEEK material that shows PEEK can significantly lessen the risks of cage subsidence into the vertebral body [4]. The majority of the previous studies [5]-[7] has confirmed that PEEK cages could significantly improve fusion rates (93-100\% in 12 months' time postoperatively), reduce subsidence rates on cage-endplate interfaces $(0-14.2 \%)$ and exhibit excellent clinical outcomes (80-96\%).

The used of bilateral cage with PI has shown to be the perfect method to achieve high segmental stability. However, this condition is riskier and costly. On these regards, a single 
spacer might cost $\$ 2,000-5000$ and bilateral cage insertion required wider distraction of facetectomy and laminectomy which increases the risk of neurologic damage due to major nerve root manipulation [8]. Based on this basis, cost effective, less risky and speedy recovery are the ultimate choices for whosever seeking interbody fusion surgery. Therefore, there is a strong demand in promoting the usage of unilateral cage in interbody fusion surgery. Unilateral cage oriented in oblique position has been reported to have identical potential as bilateral cage did. Moreover, this configuration could also significantly reduce cage-endplate interface stress, provide better segmental stability, and demonstrate less pedicle screw stress [9]-[11]. To further reduce the cost of the treatment, there was an attempt to adopt unilateral pedicle screw and rod constructs in either unilateral or bilateral cage fusion. However, according to Ambati et al. [9], this effort is ineffective because it would greatly undermine the construct stability that lead to a construct failures such as screw loosening due to repetitive loading conditions.

This study was formulated to quantitatively assess the efficiency of unilateral PEEK spacer with bilateral PI oriented in an oblique positioned by comparing its biomechanical performance with other set of PLIF constructs (different cage material and orientation) of the following: bilateral PEEK; bilateral titanium; and unilateral titanium plus oblique orientation. To achieve this goal, finite element analysis (FEA) approach was utilized to scrutinize the cage subsidence symptom and pedicle screw loosening effect by means of stress distribution patterns.

\section{MATERIALS AND METHODS}

A three dimensional (3D) model of L4 to L5 lumbar segment was created form a CT scan data of a patient via MECHANICAL FINDER ${ }^{\mathrm{TM}}$ software (Research Center of Computational Mechanics Co. Ltd. Japan). The CT scan images were obtained from a 29-year-old Japanese male subjects without any previous medical history. The weight and height of the patient was $78 \mathrm{Kg}$ and $176 \mathrm{~cm}$, respectively. Written informed consent was obtained prior to participation in the research.

The vertebral bodies were assigned as a cancellous bone core surrounded by a $0.4 \mathrm{~mm}$ thick cortical shell. The cancellous bone, intervertebral discs and facet joint cartilages were modelled with $1.0 \mathrm{~mm}$ solid tetrahedral element while the cortical bone was modelled with $1.0 \mathrm{~mm}$ linear shell triangular element. The bone material properties were defined as non-linear and inhomogeneous material. To reflect the heterogeneity of bone, the Young's modulus and yield strength for each of the bone element were formulated based on the Hounsfield Unit (HU) values of the CT scan images in accordance to the relationship as stated by Keyak et al. [12]. The Poisson's ratio for the bones was fixed at 0.4 [12]. In contrast, the intervertebral discs and facet joint cartilages were defined as linear and homogeneous material. The intervertebral discs Young's modulus (E) and Poisson's ratio were set at $8.4 \mathrm{MPa}$ and 0.45 , while for the facet joint they were set at $11 \mathrm{MPa}$ and 0.2 , respectively [13-14]. Any relative movements of the intervertebral disc and facet joint cartilages were restrained by introducing perfectly bonded interface condition to the vertebral bodies.

Two commercially available interbody fusions (Telamon $^{\mathrm{TM}}$, Medtronic Sofamor Danek, Memphis, TN) fabricated from titanium $(10 \mathrm{~mm}$ in height, $22 \mathrm{~mm}$ in length, and $10 \mathrm{~mm}$ in width) and PEEK ( $8 \mathrm{~mm}$ in height, $26 \mathrm{~mm}$ in length, and 10 $\mathrm{mm}$ in width) materials were utilised and simulated in this study. $40 \%$ of the laminae and spinous processes of L4 were removed in order to simulate the spacer placement into the $3 \mathrm{D}$ model. The cages were inserted into the disc space in either unilateral or bilateral configuration. For the bilateral cage fusion, the cages were positioned symmetrically at the midsagittal plane, while for the unilateral cage fusion, the cage was placed in an oblique position. The cages were securely attached to intervertebral disc to inhibit any relative movement during the simulation. Next, a bilateral PI was instrumented to the model by inserting pedicle screws (51.8 $\mathrm{mm}$ in length and $6.2 \mathrm{~mm}$ in diameter) and rods $(50 \mathrm{~mm}$ in length) to the vertebral bodies. The pedicle screws-rods system was assigned with Titanium material properties. The contact surfaces of the pedicle screws-rods and pedicle screws-bone were simplified to be in perfectly bonded.

Finally, four PLIF constructs shown in Figure 1 with different type of cage orientations were developed. The models were loaded with five physiological spine motions namely; compression $(1000 \mathrm{~N})$, extension $(1.0 \mathrm{Nm})$, flexion $(4.2 \mathrm{Nm})$, lateral bending $(2.6 \mathrm{Nm})$ and axial rotation $(3.4 \mathrm{Nm})$ [15]. The loads were applied to the intervertebral disc located immediately on the top of L4 (85\% of the load) and to the superior facet joint cartilages attached to L4 (15\% of the load) based on three column load bearing concept [16]. The intervertebral disc located below L5 and the inferior facet joint cartilages attached to L5 were fixed in all directions.

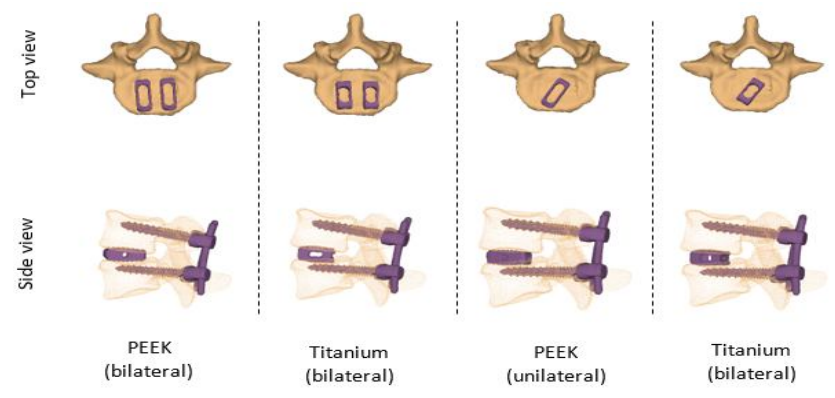

Figure 1: Simulated PLIF Construct 


\section{RESSULTS AND DISCUSSIONS}

\subsection{Cage-Endplate Interface Stress}

Figure 2 shows the Drucker-Prager stress distributions of the different PLIF constructs under the application of spine physiological motions. In order to further corroborate the stress distribution patterns on the vertebral bodies of each model as shown in Figure 3, 20 area of interests (AoIs) were selected to determine the maximum value of the stress. Obviously, in overall the highest Drucker-Prager stresses for each of the spine motion (except in lateral bending motion) were produced by obliquely-placed unilateral titanium cage construct. In contrast, the lowest Drucker-Prager stresses were mostly generated by obliquely-placed unilateral PEEK cage construct. In addition, it is important to note that they were also exhibited the similar stress profiles in all motion activities.

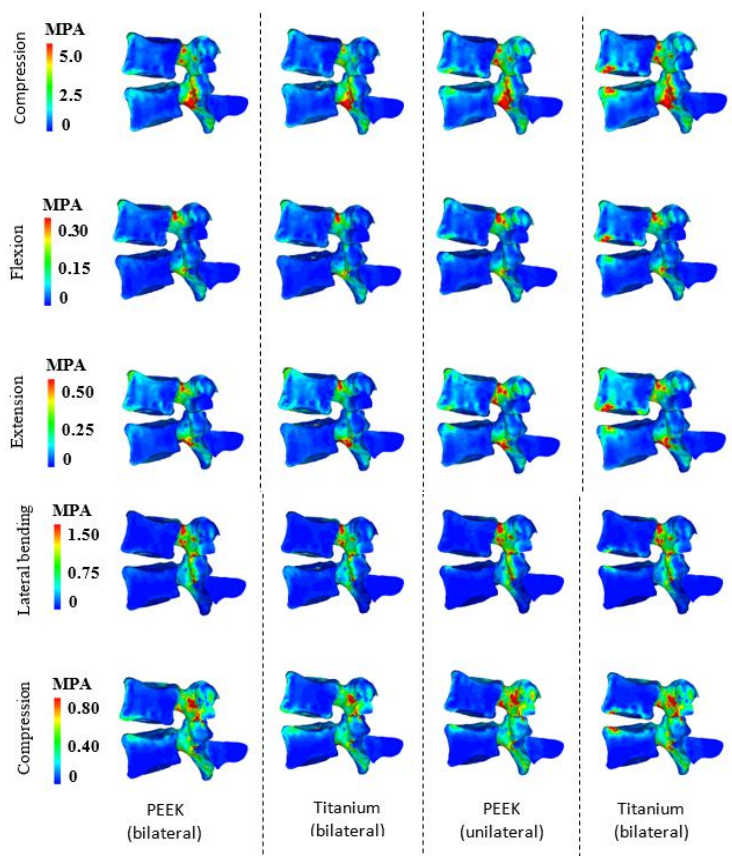

Figure 2: Drucker-Prager Stress Distributions (Sagittal Views)

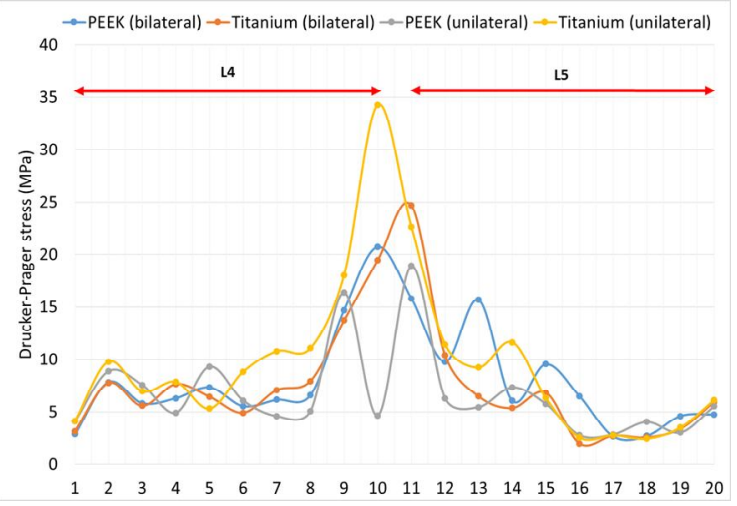

(a)

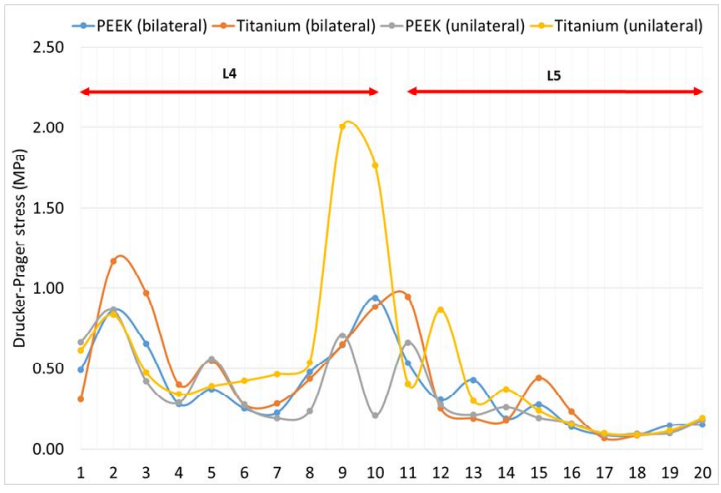

(b)

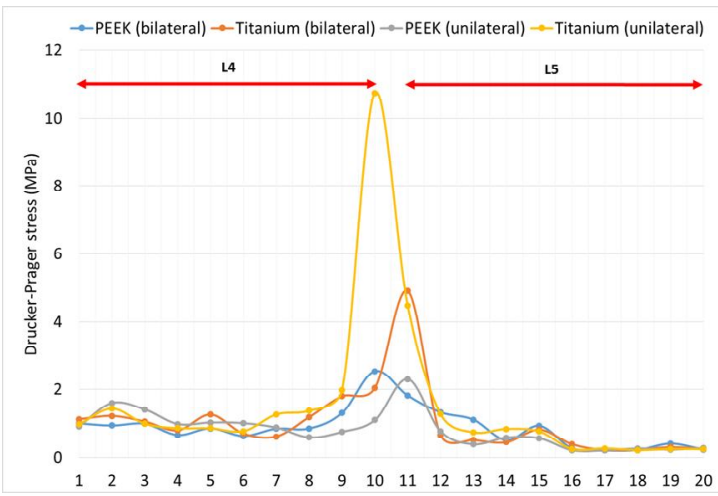

(c)

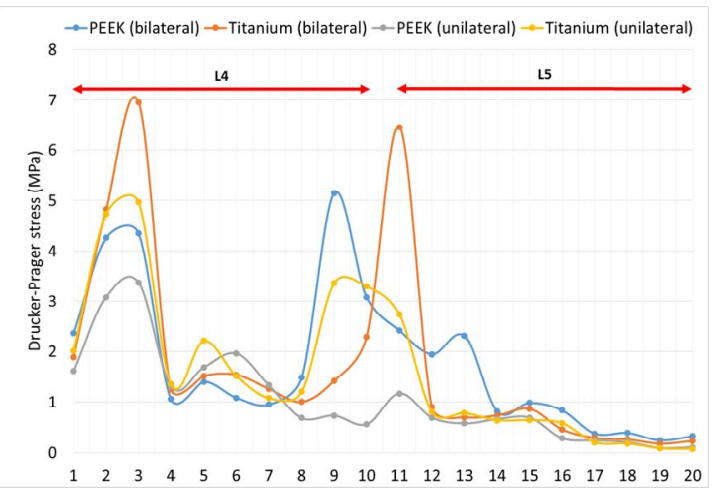

(d)

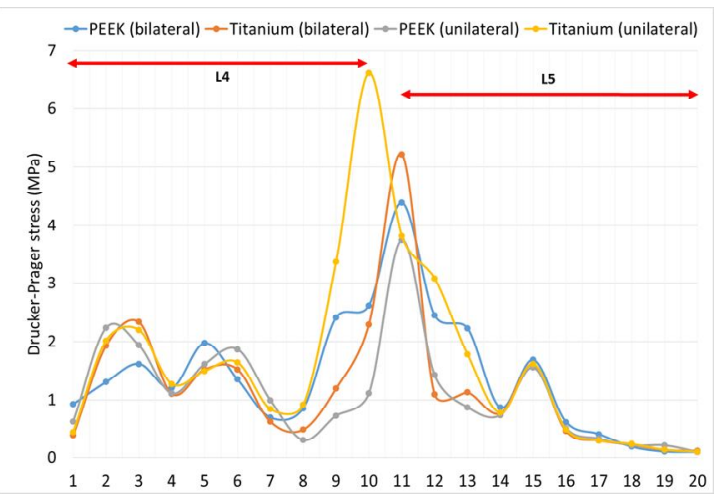

(e)

Figure 3. Maximum Drucker-Prager Stress Distributions Obtained during (a) compression, (b) flexion, (c) extension, (d) lateral bending and (e) axial rotation. 
Apparently, in almost all of the specified spine motions, we have found that the highest stress generations were produced at the cage-endplate interface junctions. The existence of this spike stress disturbance at this contacting area could be classified as a distortion stress due to the application of the cages into the intervertebral disc, which is more likely due to stiffness mismatch between the cage and the vertebral bone. Based on this criterion, the maximal Drucker-Prager stress distributions at the cage-enplate junction are summarized into a bar graph as illustrated in Figure 4. The distortion stresses ranged from 1.11 to $20.72 \mathrm{MPa}, 1.00$ to $24.67 \mathrm{MPa}, 0.73$ to 18.9 MPa and 2.01 to 38.19 MPa for the bilateral PEEK cage, bilateral titanium cage, obliquely-placed unilateral PEEK cage and obliquely-placed unilateral titanium cage construct, respectively. Based on these figures, the cage subsidence symptom was highly affected the obliquely-placed unilateral titanium cage followed by bilateral titanium cage, bilateral PEEK and obliquely-placed unilateral PEEK cage, accordingly.

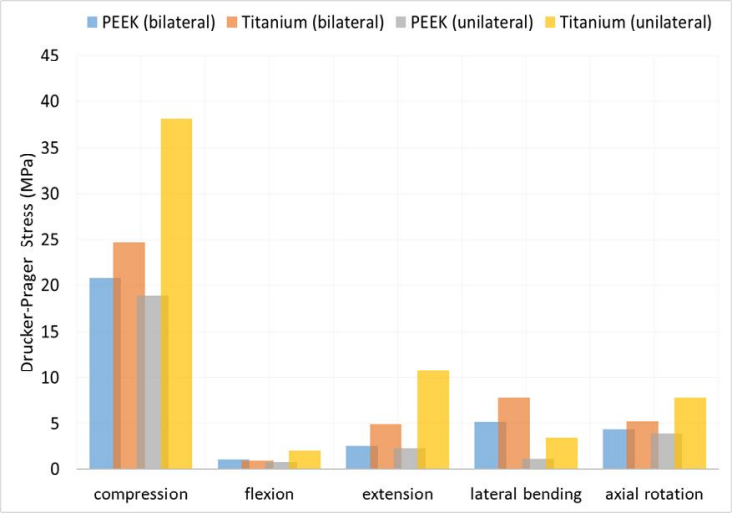

Figure 4: Cage-Endplate Interface Stress

Apparently, the selection of the cage material plays the utmost significance in eradicating the cage subsidence impact rather than the cage orientation. However, this finding has drawn a unique conclusion that for a material having a close proximity to that of cortical bone material properties, the obliquely-placed cage is the best practice. However, for the stiffer material the bilateral configuration is the most practical way.

\subsection{Bone-Pedicle Screw Interface Stress}

The effect of pedicle screw loosening was carefully investigated via maximal bone-pedicle screws interface stress as depicted in Figure 5. Obviously, the most significance load bearing variations occurred during compression activity. In other motions, they became incomparable because of their relative differences and maximal values are less than $10 \%$ and $10 \mathrm{MPa}$, respectively. Therefore, based on this condition it was cleared to us that the obliquely-placed unilateral titanium cage construct has the greatest tendency to increase the load bearing on PI construct (87.1 MPa). This was then consecutively followed by bilateral titanium cage (69.1 MPa), obliquely-placed PEEK cage (68.5 MPa) and bilateral PEEK cage construct $(61.7 \mathrm{MPa})$. Certainly, double spacer configuration shows better load dispersion on the bone-pedicle screws interfaces irrespective of the type of the material. However, if the type of the cage material is considered, likewise the higher stress profiles would be detected on the stiffer material (titanium) regardless of its number and cage configuration.

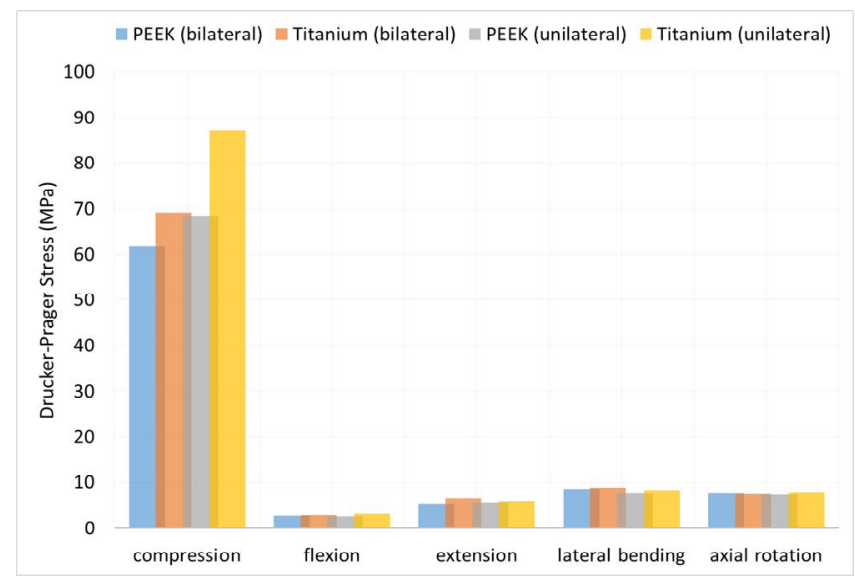

Figure 5: Bone-Pedicle Screw Interface Stress

\subsection{Relative Stress Concentration Gap Difference}

The relative stress concentration gap difference between the cage-endplate and bone-pedicle screw interface in reference to the stress generated on the bone-pedicle screw interface was used to investigate the stress mitigation impact on the cage-endplate interface. The relative stress concentration difference for the unilateral PEEK, bilateral PEEK, bilateral titanium and unilateral titanium cage construct was $72 \%, 66 \%, 64 \%$ and $56 \%$, respectively. These results suggest that the PEEK-based cage construct could significantly reduce the load-bearing distribution afflicted on the anterior part of the vertebral body with the obliquely-positioned cage construct showed the most excellent endplate stress mitigator. Furthermore, the high magnitude indicates a large amount of load would be transferred through the PI, reducing the stress concentration on the cage-endplate interface and thus reducing the risk of cage subsidence.

\subsection{Discussion}

The results have shown that the application of PEEK material could significantly reduce the stress shielding effect and has better load sharing mechanisms. These conditions were reflected by the least amount of stress generated by the PEEK compared to the titanium cage at the both endplate and facet joint junction. Notably, almost all of the maximal interface 
stresses were generated below the average of the normal people bone's yield strength (83 MPa) [17] except at the bone-pedicle screw interface for the obliquely-placed titanium cage. This condition indicates that only unilateral titanium cage construct was at the higher risk of screw loosening effect.

From this finding, we conclude that the titanium-based spacer is not recommended to be implanted in osteoporosis patient. This is because this condition will further deteriorate the stress shielding effect and hence will undermine the structural integrity of the reconstructive configuration by putting the bone at higher risks of bone fracturing even by performing basic daily living activity. FEA study conducted by Adam et al. [2] has shown that the simulated severe osteoporosis in vertebral body model has caused the endplate stress increases up to three-fold than its initial state. Hence, low stiffness accompanied by high ultimate and tensile strength [6] are the remarkable benchmark to describe the applicability and biocompatibility of the PEEK-based cage in PLIF surgery.

Technically, the smaller stress in the PEEK spacer the lesser the subsidence effect will be detected at the endplate junctions. This is most likely due to the higher stress that has been effectively transferred through the stiff structure of the rod and pedicle screw system which has been proven by the higher relative stress difference between the posterior and the anterior column of the vertebral body. This is in tandem with the purpose of instrumenting PI in order to provide structural support to the spine. This is based on the fact that this point of attachment is very crucial in terms of transferring and supporting load between the pedicle screws and the vertebral bones [14] so that the load could be transferred to the adjacent vertebral body in a safe and effective manner. It has been reported that the application of PI could significantly reduce the cage-endplate interface stress by 50 to $60 \%$ if compared to non-instrumented cage [1]. In addition, the application of PI would also mitigate the risks of cage failure and cage migration by increasing the cage pullout resistance [9][10][18]. Even though it seems like the increased stress on the pedicle screw would increase the load burden on that area, however we have to bear in mind that the stress generation by the PEEK-based cage was comparably lower than the stress generation produced by titanium-based cage construct.

\section{CONCLUSIONS}

The performance of the mechanical and clinical outcomes in PLIF surgery procedures lies concretely within the type of material used as a cage material and the orientation of the cage configuration. Indeed, obliquely-placed unilateral PEEK spacer with PI offers greater immediate stability as well as reduces cage subsidence and pedicle screw loosening symptoms. Therefore, this surgical technique could be utilized as an alternative to bilateral cage fusions by mitigating the surgical costs and lessen the risks of construct failure and spinal injuries. Continued analyzation along with clinical experimentation will be required to achieve further refinement on the applicability of the reconstructive configuration.

\section{ACKNOWLEDGEMENT}

This research was supported by Universiti Tun Hussein Onn Malaysia, under TIER1-H118. We thank and acknowledge our colleagues from Kyushu University and Juntendo University Japan who provided insight and expertise that greatly assisted the research.

\section{REFERENCES}

1. Y. H. Tsuang, Y. F. Chiang, C. Y. Hung, H. W. Wei, C. H. Huang, and C. K. Cheng, Comparison of cage application modality in posterior lumbar interbody fusion with posterior instrumentation-A finite element study, Med. Eng. Phys., vol. 31, no. 5, pp. 565-570, 2009.

2. C. Adam, M. Pearcy, and P. McCombe, Stress analysis of interbody fusion-finite element modelling of intervertebral implant and vertebral body, Clin. Biomech., vol. 18, no. 4, pp. 265-272, 2003,

3. S. M. Kurtz and J. N. Devine, PEEK biomaterials in trauma, orthopedic, and spinal implants, Biomaterials, vol. 28, no. 32. pp. 4845-4869, 2007.

4. R. J. Mobbs, A. M. T. Chau, and D. Durmush, Biphasic calcium phosphate contained within a polyetheretherketone cage with and without plating for anterior cervical discectomy and fusion., Orthop. Surg., vol. 4, no. 3, pp. 156-65, 2012.

5. M. Cabraja, S. Oezdemir, D. Koeppen, and S. Kroppenstedt, Anterior cervical discectomy and fusion: comparison of titanium and polyetheretherketone cages., BMC Musculoskelet. Disord., vol. 13, p. 172, 2012.

6. C.-C. Niu, J.-C. Liao, W.-J. Chen, and L.-H. Chen, Outcomes of interbody fusion cages used in 1 and 2-levels anterior cervical discectomy and fusion: titanium cages versus polyetheretherketone (PEEK) cages., J. Spinal Disord. Tech., vol. 23, no. 5, pp. 310-6, 2010.

7. E. Chong, M. H. Pelletier, R. J. Mobbs, and W. R. Walsh, The design evolution of interbody cages in anterior cervical discectomy and fusion: a systematic review., BMC Musculoskelet. Disord., vol. 16, p. 99, 2015.

8. R. W. Molinari, J. Sloboda, and F. L. Johnstone, Are 2 cages needed with instrumented PLIF? A comparison of 1 versus 2 interbody cages in a military population., Am. J. Orthop. (Belle Mead. NJ)., vol. 32, no. 7, pp. 337-43; discussion 343, 2003.

9. A. D.V. et al., Bilateral pedicle screw fixation provides superior biomechanical stability in transforaminal lumbar interbody fusion: A finite 
element study, Spine J., vol. 15, no. 8, pp. 1812-1822, 2015.

10. S.-T. Wang et al., Posterior instrumentation reduces differences in spine stability as a result of different cage orientations: an in vitro study., Spine (Phila. Pa. 1976)., vol. 30, no. 1, pp. 62-7, 2005.

11. M.-F. Chiang, Z.-C. Zhong, C.-S. Chen, C.-K. Cheng, and S.-L. Shih, Biomechanical comparison of instrumented posterior lumbar interbody fusion with one or two cages by finite element analysis., Spine (Phila. Pa. 1976)., vol. 31, no. 19, pp. E682-9, 2006.

12. J. H. Keyak, S. A. Rossi, K. A. Jones, and H. B. Skinner, Prediction of femoral fracture load using automated finite element modeling., J. Biomech., vol. 31, no. 2, pp. 125-33, 1998.

13. M. Alizadeh et al., The use of $\mathbf{X} \square$ shaped cross $\square$ link in posterior spinal constructs improves stability in thoracolumbar burst fracture: A finite element analysis, J. Orthop. Res., vol. 31, no. 9, pp. 1447-1454, 2013.

14. D. Tawara, K. Noro, T. Tsujikami, Y. Okamoto, and H. Murakami, Nonlinear mechanical analysis of posterior spinal instrumentation for osteoporotic vertebra: Effects of mechanical properties of the rod on the failure risks around the screw, J. Biomech. Sci. Eng., vol. 9, no. 2, 2014.

15. K.-S. Han, A. Rohlmann, T. Zander, and W. R. Taylor, Lumbar spinal loads vary with body height and weight., Med. Eng. Phys., vol. 35, no. 7, pp. 969-77, 2013.

16. O. PEREY, Fracture of the vertebral end-plate in the lumbar spine; an experimental biochemical investigation, Acta Orthop. Scand. Suppl., vol. 25, pp. $1-101,1957$.

17. F. Gaynor Evans, The Mechanical Properties of Bone, Artif. Limbs, vol. 13, no. 1, pp. 37-48, 1969.

18. M. H. Jalil, M. H. Mazlan, and M. Todo, Biomechanical Comparison of Polymeric Spinal Cages Using Ct Based Finite Element Method, Int. J. Biosci. Biochem. Bioinforma., vol. 7, no. 2, pp. 110-117, 2017. 